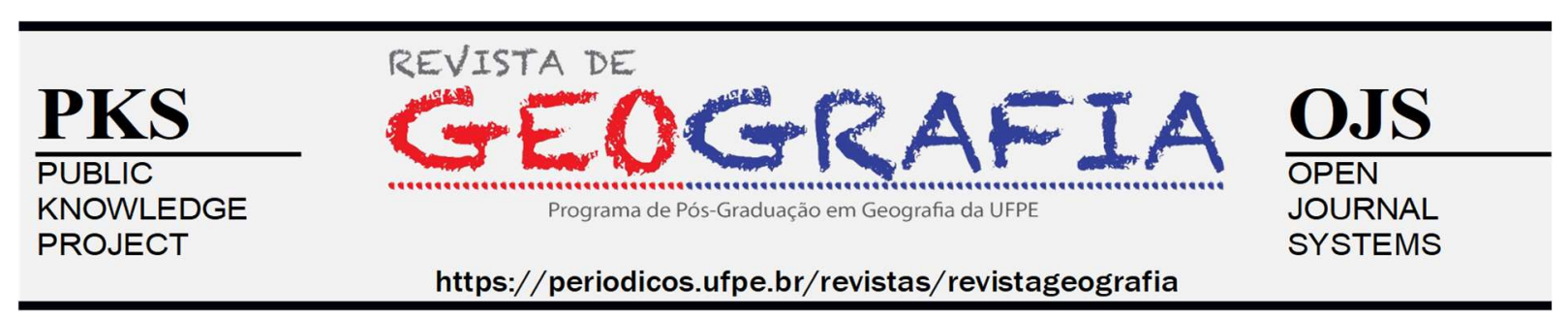

\title{
AVALIAÇÃO DE MÉTODOS DE INTERPOLAÇÃO ESPACIAL APLICADOS À ESTIMATIVA DE CHUVA EM REGIÃO AMAZÔNICA: BACIA DO RIO MADEIRA
}

\author{
Vinicius Alexandre Sikora de Souza ${ }^{1}$, Otto Corrêa Rotunno Filho ${ }^{2}$, Daniel Medeiros Moreira ${ }^{3}$ \\ ${ }^{1}$ Universidade Federal do Rio de Janeiro.E-mail: vass1000@gmail.com \\ ${ }^{2}$ Professorda UFRJ.E-mail: otto@coc.ufrj.br \\ ${ }^{3}$ Engenheiro da CPRM. E-mail: daniel.moreira@cprm.br
}

Artigo recebido em 17/05/2019 e aceito em 25/12/2019

\begin{abstract}
Resumo: O presente trabalho objetiva avaliar comparativamente diferentes métodos de interpolação espacial de precipitação para a bacia hidrográfica do rio Madeira, ao longo do ano de 2011, mediante aquisição de dados tradicionalmente coletados na escala do pluviômetro. Foram usadas 60 estações, onde se realizaram interpolações a partir de 45 delas (70\%), deixando o conjunto restante de 15 (30\%) para validação do desempenho dos métodos adotados. Os procedimentos de espacialização envolveram (i) média aritmética (MA), (ii) polígonos de Thiessen (PT), (iii) função de base radial multiquadrada (BRM), (iv) krigagem (KG), (v) inverso da distância ponderada (IDP) e (vi) vizinho natural (VN). Verificou-se que os melhores desempenhos foram atingidos pelos métodos de krigagem e de inverso da distância ponderada, com certo grau de similaridade com os resultados obtidos pela média aritmética, enquanto o método da função de base radial multiquadrada (BRM) apresentou o pior resultado quando comparado com os demais procedimentos aplicados. O estudo permitiu concluir que a atual rede pluviométrica na bacia do rio Madeira é extremamente limitada para bem caracterizar a variabilidade espaçotemporal da chuva na região.
\end{abstract}

Palavras-chaves: Chuva; Interpoladores espaciais; Região Amazônica

\section{EVALUATION OF SPATIAL INTERPOLATION METHODS APPLIED TO RAINFALL ESTIMATION IN THE AMAZON REGION: MADEIRA RIVER BASIN}

Abstract: This work has the purpose of evaluating comparatively different and commonly used precipitation interpolation methods for the Madeira river basin employing spatial analysis based on traditional rainfall data collected at the raingauge scale along the year of 2011. Sixty stations were used, where 45 stations $(70 \%)$ were used for interpolation and the remaining $15(30 \%)$ were left for evaluating the performance of the adopted methods. The procedures analyzed were (i) arithmetic mean (MA), (ii) Thiessen polygons (PT), (iii) multifaced radial base function (BRM), (iv) kriging (KG), (v) inverse distance weighting (IDP) and (vi) natural neighbor (VN). It was verified that the best performances were achieved by the methods of kriging and inverse distance weighting with a certain degree of equivalence to the arithmetic mean approach, while the multi-square radial base function (BRM) method presented the worst results when compared to the other methods applied. The study allowed to conclude that the current raingauge network installed in the Madeira river basin is severely limited to characterize the spatiotemporal variability of rainfall in the region

Key-words: Rainfall; Spatial Interpolators; Amazon Region 


\section{INTRODUÇÃO}

A precipitação, dentre os elementos hidroclimatológicos, é o que mais interfere na vida humana, uma vez que se configura como a principal entrada de água no sistema hidrológico, tornando outras variáveis, como a vazão e a infiltração, intimamente ligadas à sua ocorrência. Em virtude de sua larga influência sobre áreas povoadas, por vezes, percebida com perspectiva positiva, enquanto, em outras, negativa, a chuva pode ser considerada a principal fonte de suprimento de corpos hídricos que permitem o desenvolvimento das diversas atividades humanas e econômicas de nossa sociedade (ALMEIDA et al., 2011).

No Brasil, as informações de volumes precipitados são disponibilizadas em grande parte pela Agência Nacional das Águas em forma de séries históricas no sistema de informações hidrológicas HidroWeb. Por outro lado, pelo menos duas dificuldades limitam os trabalhos de entendimento do fenômeno.

A primeira refere-se à densidade irregular das estações pluviométricas que possuem, em muitas regiões, pouca representatividade espacial devido ao baixo número de estações pluviométricas e pela grande distância entre elas. Segundo Clarke e Dias (2002), a distribuição das estações pluviométricas é mais densa nos estados altamente urbanizados (São Paulo - uma estação a cada $169 \mathrm{~km}^{2}$; Distrito Federal - uma estação a cada $166 \mathrm{~km}^{2}$; Paraná: uma estação a cada $214 \mathrm{~km}^{2}$ ); em contraste, o Amazonas tem uma estação a cada $7.829 \mathrm{~km}^{2}$, enquanto Pará apresenta uma estação a cada $5.528 \mathrm{~km}^{2}$ e Mato Grosso uma estação a cada 4.637 km².

A segunda dificuldade surge pelo grande número de dados faltantes nas séries históricas, situação gerada pela ausência do observador, falhas nos mecanismos de registro, perda das anotações ou das transcrições dos registros pelos operadores e encerramento das observações (STRECK et al., 2009).

Como possível solução ou superação desses obstáculos, Conti (2002) destaca, entre outros, a possibilidade do uso dos métodos de média aritmética de postos presentes na bacia, de média ponderada pelas áreas abrangidas pelos polígonos de Thiessen e de geração de isoietas por interpolação dos dados pluviométricos.

$\mathrm{O}$ método da média aritmética (MA) configura-se na suposição de que todos os pluviômetros possuam o mesmo grau de abrangência na área em que se encontram, ou seja, que exerçam sua influência na precipitação média de forma similar. Logo, um dado faltante em uma 
estação será aproximadamente igual à média dos dados nas estações localizadas em sua proximidade (TUCCI, 2009).

O método de Thiessen (PT), por sua vez, considera que cada posto pluviométrico possuirá uma área de influência dentro do perímetro em que se encontra. Tal procedimento prevê que tais postos estão dispostos não uniformemente no espaço. A hipótese é que a falha em uma estação pluviométrica pode ser preenchida por valor similar àquele da área de influência do posto com dados na qual está inserida (TUCCI, 2009).

A interpolação é um procedimento que permite construir um novo conjunto de dados a partir de um arranjo discreto de informações pontuais conhecidas, ou seja, possibilita a reconstituição aproximada de uma função apenas conhecendo algumas de suas abscissas (domínio) e respectivas ordenadas (imagem) (TUCCI, 2009). Permite estimar um valor para o qual não existe informação disponível, considerando-se que os pontos próximos no espaço tendem a ter valores mais semelhantes do que pontos mais afastados. Os interpoladores mais empregados em chuvas, segundo Righi e Basso (2016), são a função de base radial multiquadrada (BRM), o método de krigagem (KG), o inverso da distância ponderada (IDP) e o vizinho natural $(\mathrm{VN})$.

Nesse contexto, o uso dos operadores de média aritmética, de polígonos de Thiessen e dos interpoladores função de base radial multiquadrada (BRM), método de krigagem (KG), inverso da distância ponderada (IDP) e vizinho natural (VN) poderia suprir a carência de dados em localidades que possuem séries pluviométricas com falhas ou que dispõem de baixa densidade de estações, tal como a Amazônia, que é uma região com dados esparsos, em que as variáveis climáticas são insuficientemente quantificadas, bem como incertezas significativas permanecem no entendimento dos diferentes processos subjacentes aos mecanismos termodinâmicos e dinâmicos do clima associados à variabilidade em uma ampla variação de escalas temporais e espaciais

Desse modo, o objetivo deste estudo foi avaliar comparativamente diferentes métodos de interpolação de precipitação para a bacia hidrográfica do rio Madeira, por meio de análise espacial na escala mensal, ao longo do ano de 2011, período que dispõe do maior número de estações sem falhas nos registros pluviométricos observacionais. 


\section{MATERIAIS E MÉTODOS}

Área em estudo

A bacia do rio Madeira (Figura 1) situa-se na região equatorial e possui um clima quente e úmido, o que propicia a incidência de precipitações intensas e com alta variabilidade espacial, além de apresentar regime hidroclimático contrastante em diferentes porções da bacia e rios extremamente caudalosos (ESPINOZA et al., 2009).

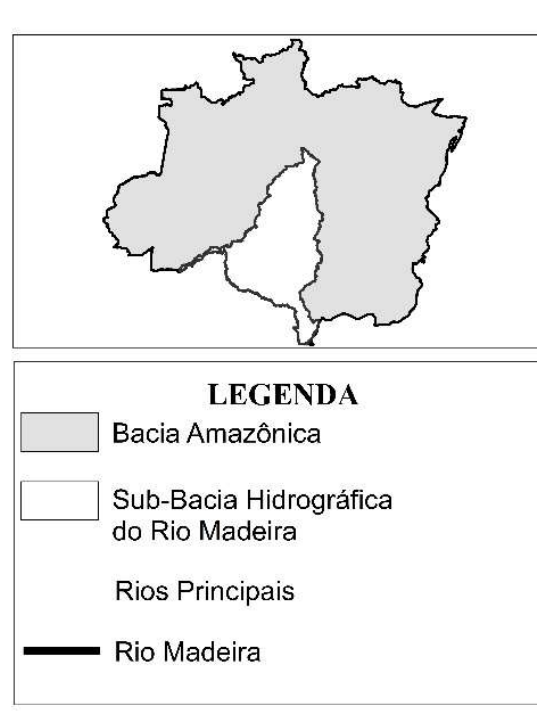

Datum: SIRGAS 2000

Fonte: Ana (2016)

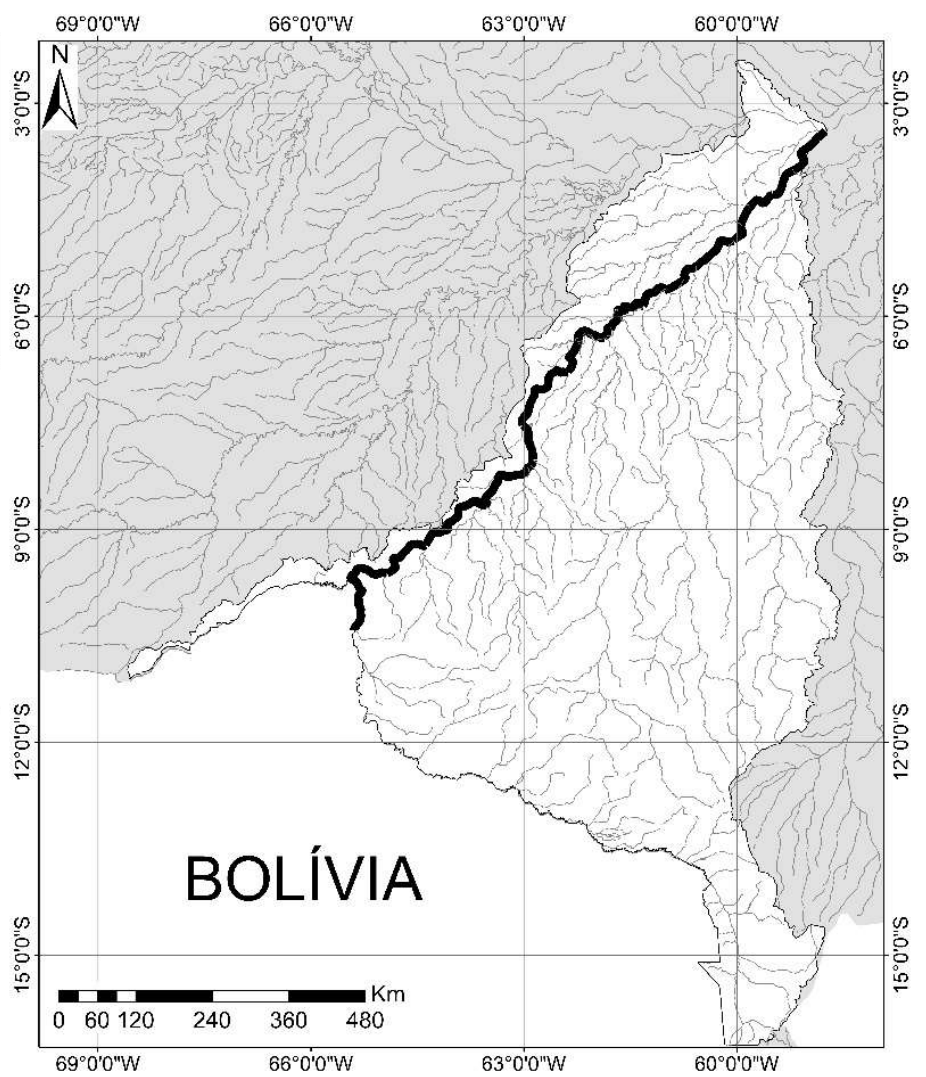

Figura 1. Localização da bacia hidrográfica do rio Madeira.

Aplicação e avaliação dos interpoladores

A seleção das informações utilizadas, neste estudo, iniciou-se pela obtenção de camada segmentada em grade de pontos incorporando todas as estações pluviométricas operadas no país no âmbito do Sistema Nacional de Informações sobre Recursos Hídricos - SNIRH da Agência Nacional das Águas - ANA. De posse da base de dados, criaram-se diagramas de Voronoi, formados por polígonos por meio de mediatrizes das linhas que conectam dois postos adjacentes e que, em seguida, são interseccionados e sobrepostos com a camada de informações Souza, Rotunno Filho e Moreira, $2020 \quad$ ISSN 0104-5490 
de dados de chuva disponíveis para a bacia do rio Madeira. Cabe observar que o procedimento é executado para se levar em conta a estimativa de estações que refletem o comportamento pluviométrico na bacia, ainda que não estejam situadas dentro de sua área.

Mediante a efetivação do procedimento anterior, chegou-se a um quantitativo de 613 estações, todavia apenas 326 possuem algum tipo de informação no banco de dados HidroWeb da Agência Nacional de Águas (ANA).

Na sequência, analisou-se o conjunto para verificar o ano de operação dotado de mais estações com registros de dados sem falhas, tendo o ano de 2011 sido selecionado por conter 60 estações, sendo 10 estações fora da área bacia e 50 internos à bacia.

Posteriormente, o conjunto das 60 estações foi separado em dois grupamentos, notadamente o grupo de calibração e o grupo de validação, conforme discriminado na Figura 2.
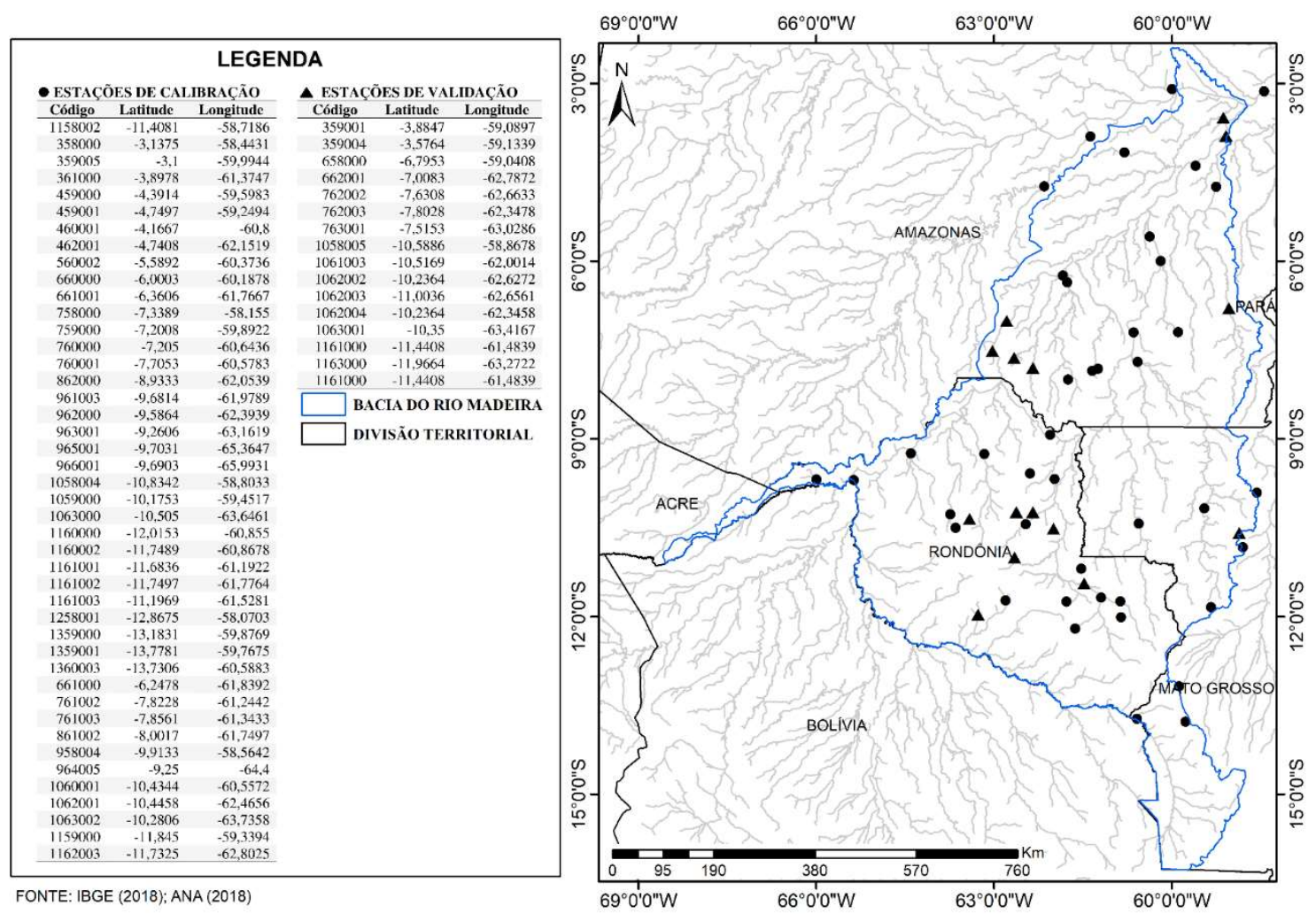

Figura 2. Estações pluviométricas dos grupos de treinamento e validação na bacia do rio Madeira.

O grupo de calibração foi composto de $70 \%$ das estações localizadas dentro da área da bacia, perfazendo 45 estações, acrescidas de 10 estações situadas fora da bacia. 
Complementarmente, observa-se que o grupo de validação foi composto de $30 \%$ das estações (15 postos pluviométricos) situadas dentro da área da bacia.

Os quantitativos acima foram estabelecidos para atender o critério do sistema de validação cruzada de Holdout (Kohavi, 1995), que foi adotado neste estudo. Tal critério exige a separação do conjunto analisado em dois subgrupos em uma proporção de aproximadamente 2/3 dos dados para treinamento (calibração) e 1/3 restante para teste (validação). Uma vez que o enfoque se concentrou na aplicação para a bacia do rio Madeira, tais proporções nas construções dos grupos foram aplicadas apenas quanto ao quantitativo de estações que estavam inseridas dentro do seu limite. Cabe ainda destacar que a formação dos grupos se deu por meio de sorteio, gerando, assim, amostras aleatórias e não dominadas por questões de subjetividade ou conveniência.

As séries históricas das estações de ambos os grupos foram acumuladas no nível mensal, gerando chuvas em unidades de mm.mês ${ }^{-1}$, posteriormente tabeladas conjuntamente com as posições geográficas de cada posto (latitude e longitude), e mais adiante exportadas em grade de pontos para o código computacional ArcGIS 10.2 (ESRI, 2017). Foram, então, aplicados, para a grade do grupo treinamento, os métodos de interpolação KG (modelo esférico), VN, IDP e BRM através da extensão “3D Analyst”, que gera campos numéricos espacializados em forma de grade a partir de dados pontuais interpolados no modo matricial, sendo que a resolução espacial adotada para a geração dos campos de precipitação foi de $0,001^{\circ}$.

Para o grupo de treinamento, foram confeccionados, ainda, os polígonos de Thiessen, usando também o código computacional ArcGIS 10.2, e determinadas as médias aritméticas mensais utilizando todos os postos desse grupo.

Na verificação da acurácia e da validação das técnicas empregadas, utilizaram-se dados do grupo de estações de validação, sendo estes últimos comparados no nível mensal por meio de análise de resíduos e do coeficiente de correlação dos dados observados dessas estações com os dados estimados por cada um dos procedimentos selecionados para a presente análise. Além disso, como índices globais de avaliação de desempenho, utilizaram-se a estimativa do erro modular médio (EMM) - Equação 1, o coeficiente de determinação $\left(\mathrm{r}^{2}\right)$ - Equação 2 e o índice de concordância (d) - Equação 3, conforme expressões respectivas abaixo dispostas como

$$
\mathrm{EPM}=\frac{\sum_{\mathrm{i}=1}^{\mathrm{n}} \sqrt{\left(\mathrm{x}_{\mathrm{E}}-\mathrm{x}_{\mathrm{M}}\right)^{2}}}{\mathrm{~N}}
$$


$r^{2}=\left(\frac{\frac{\sum_{i=1}^{n}\left(x_{M}-\overline{x_{M}}\right)\left(x_{E}-\overline{x_{E}}\right)}{n-1}}{\sqrt{\frac{\sum_{i=1}^{n}\left(x_{M}-\overline{x_{M}}\right)^{2}}{n-1} \cdot \frac{\sum_{i=1}^{n}\left(x_{E}-\overline{x_{E}}\right)^{2}}{n-1}}}\right)^{2}$

$\mathrm{d}=1-\frac{\sum_{\mathrm{i}=1}^{\mathrm{n}}\left(\mathrm{x}_{\mathrm{M}}-\mathrm{x}_{\mathrm{E}}\right)^{2}}{\sum_{\mathrm{i}=1}^{\mathrm{n}}\left[\left(\mathrm{x}_{\mathrm{E}}-\overline{\mathrm{x}_{\mathrm{M}}}\right)+\left(\mathrm{x}_{\mathrm{E}}-\overline{\mathrm{x}_{\mathrm{M}}}\right)\right]^{2}}$

onde: $\overline{\mathrm{x}_{\mathrm{E}}}-$ média de chuva estimada para o evento; $\overline{\mathrm{x}_{\mathrm{M}}}-$ média de chuva medida para o evento; $\mathrm{xE}$ - valor estimado de chuva; $\mathrm{xM}$ - valor medido de chuva; $\mathrm{N}$ - número total de estações de validação.

\section{RESULTADOS E DISCUSSÃO}

O passo inicial da análise consistiu na interpolação do acumulado mensal da série histórica de chuva do ano de 2011 (Figura 3, Figura 4, Figura 5, Figura 6 e Figura 7).

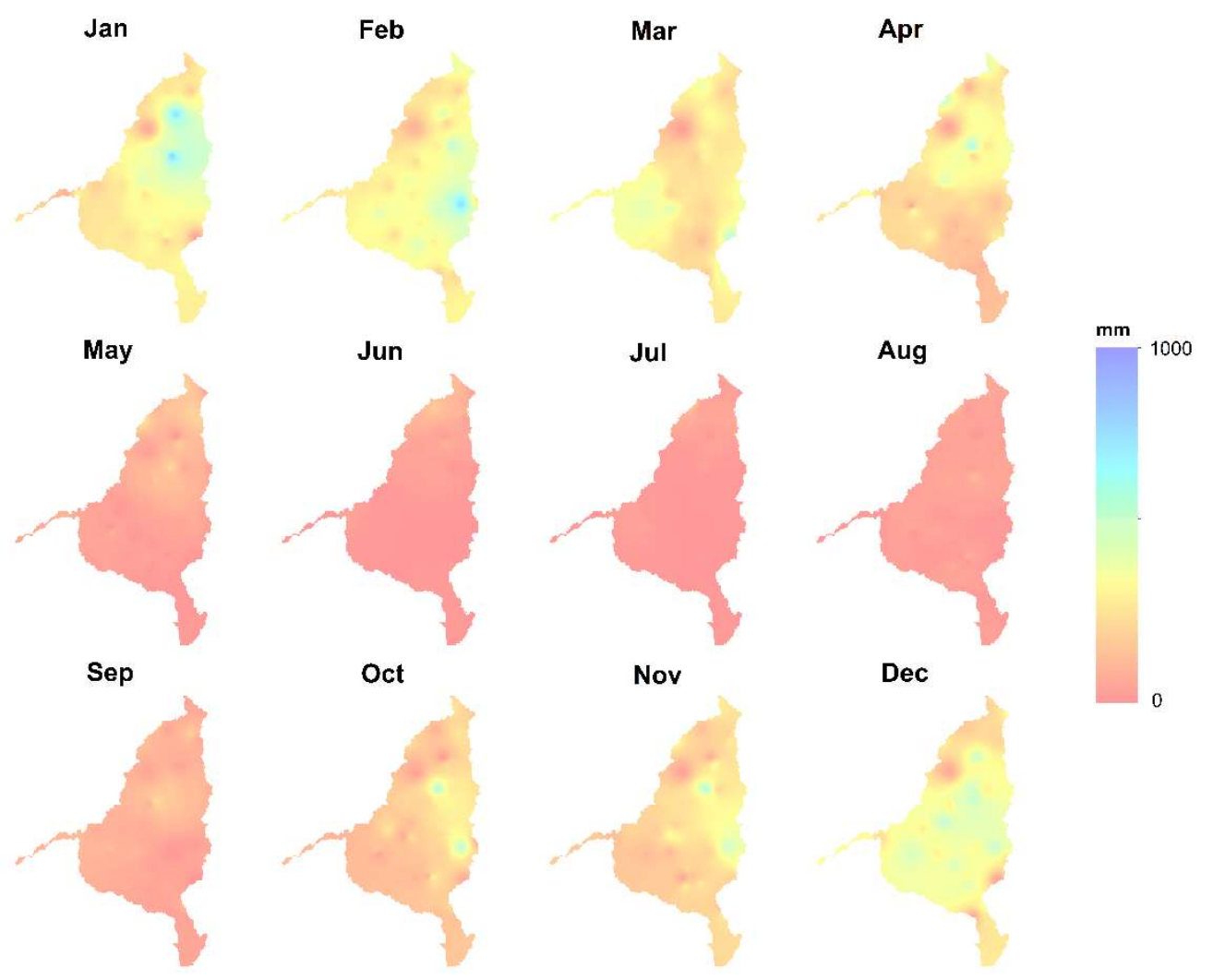

Figura 3 - Chuvas mensais (mm) interpoladas pelo IDP na bacia do rio Madeira. 


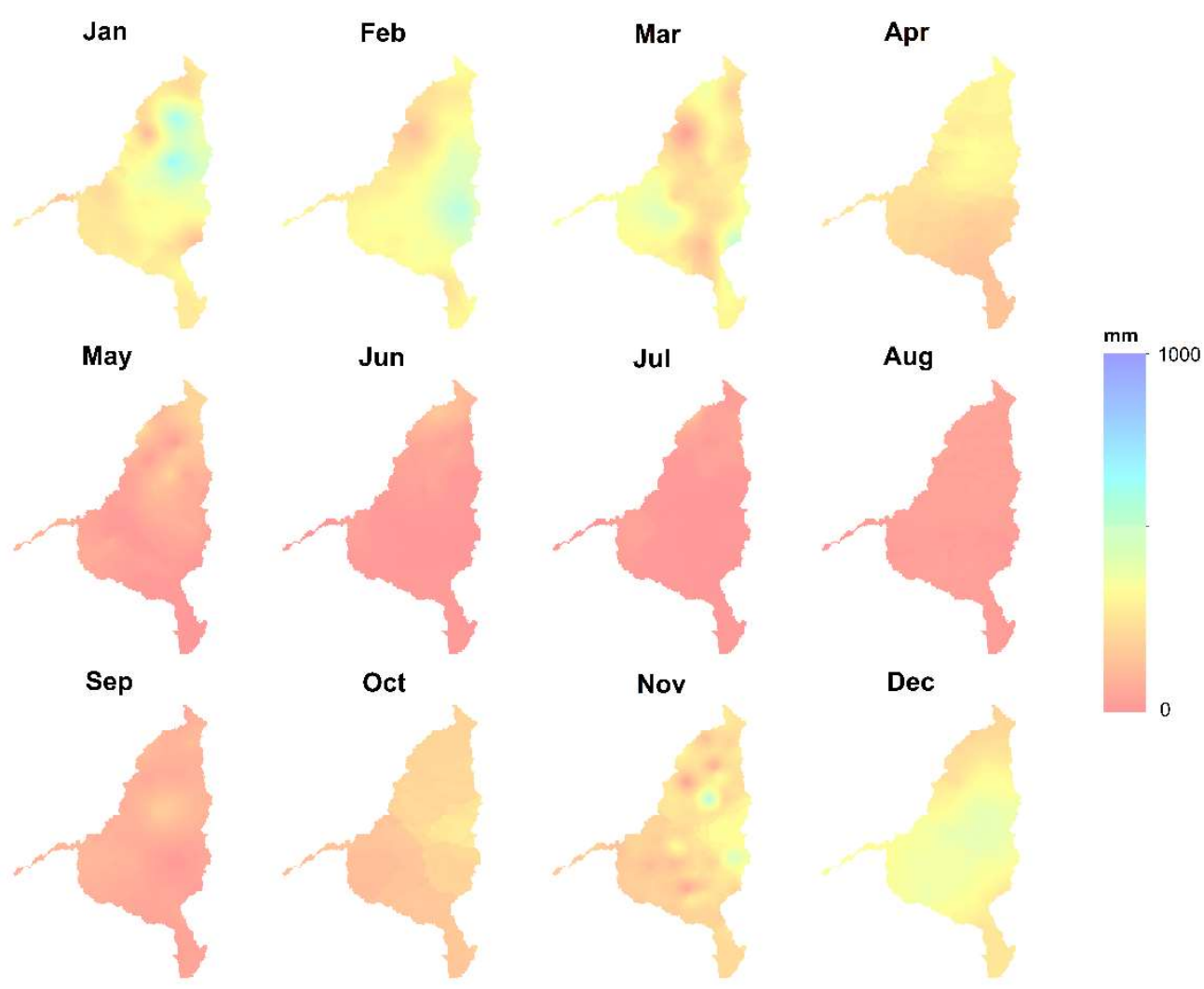

Figura 4 - Chuvas mensais $(\mathrm{mm})$ interpoladas pelo $\mathrm{KG}$ na bacia do rio Madeira.
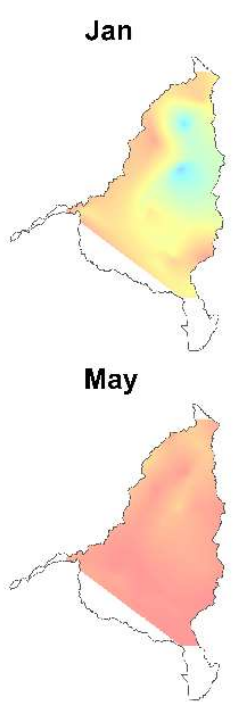

Sep

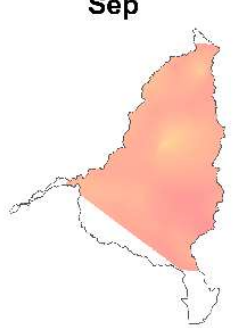

Feb

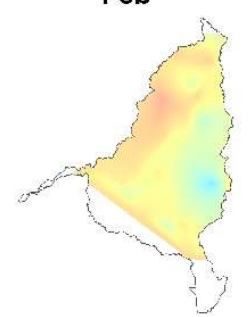

Jun

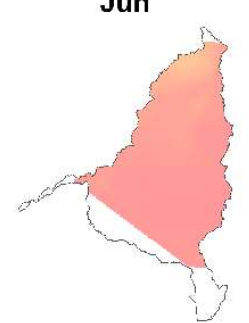

Oct

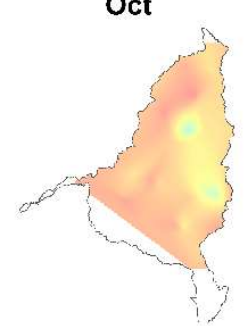

Mar

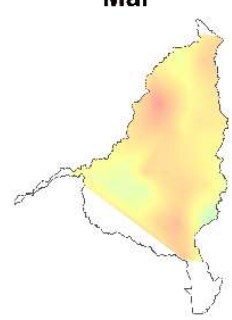

Jul

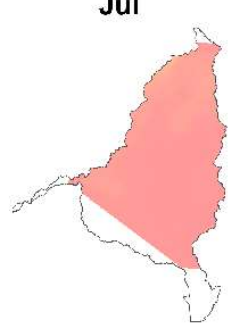

Nov

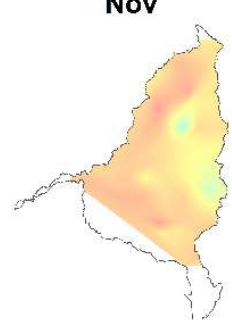

Apr
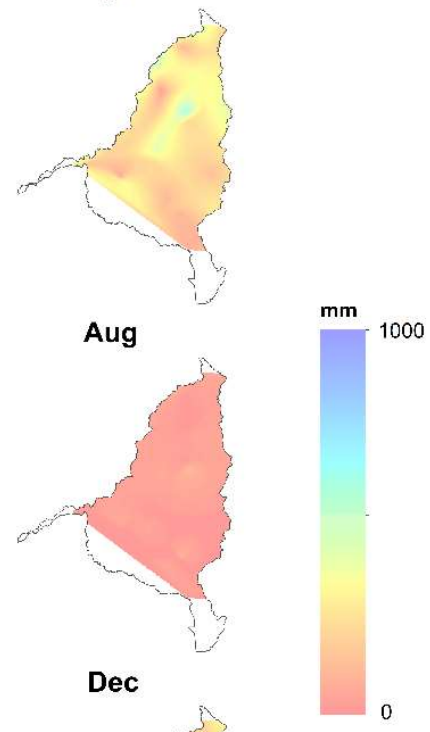

Figura 5 - Chuvas mensais $(\mathrm{mm})$ interpoladas pelo VN na bacia do rio Madeira. 


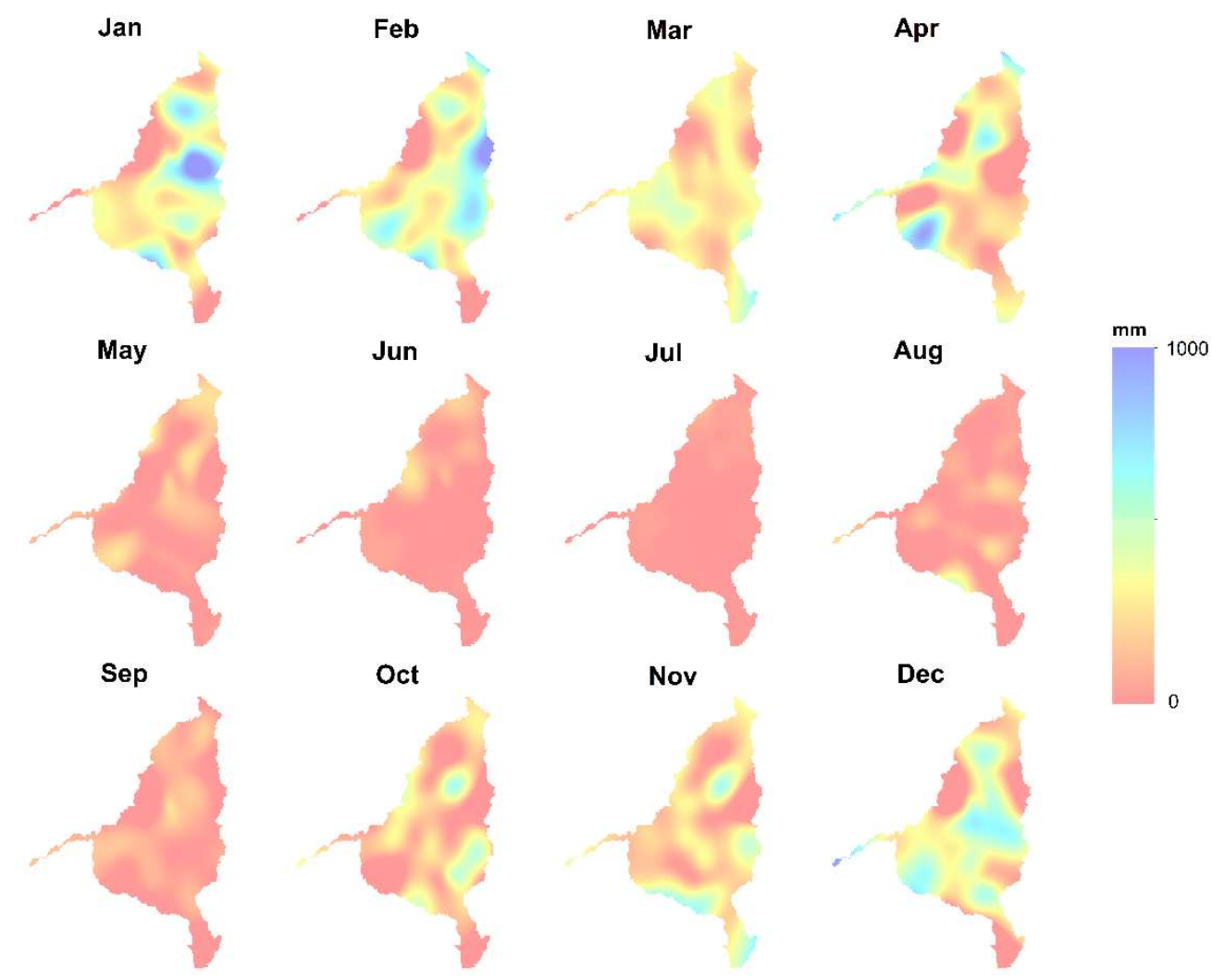

Figura 6 - Chuvas mensais ( $\mathrm{mm}$ ) interpoladas pelo BRM na bacia do rio Madeira.

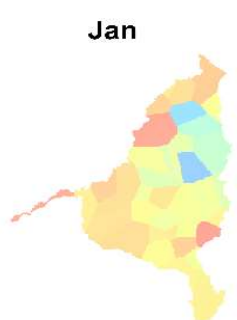

May

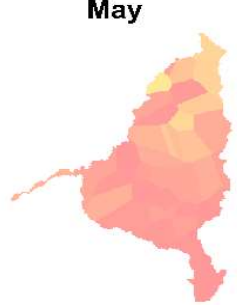

Sep
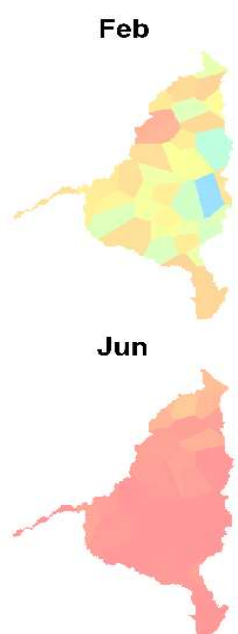

Oct

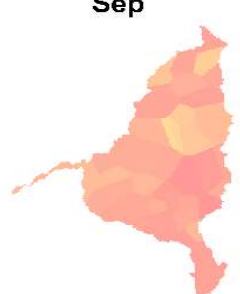

Mar

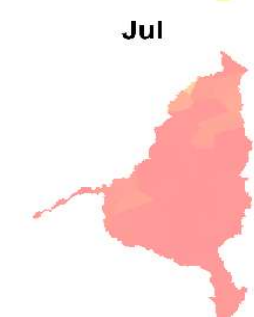

Nov
Apr

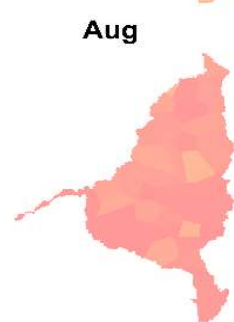

Dec
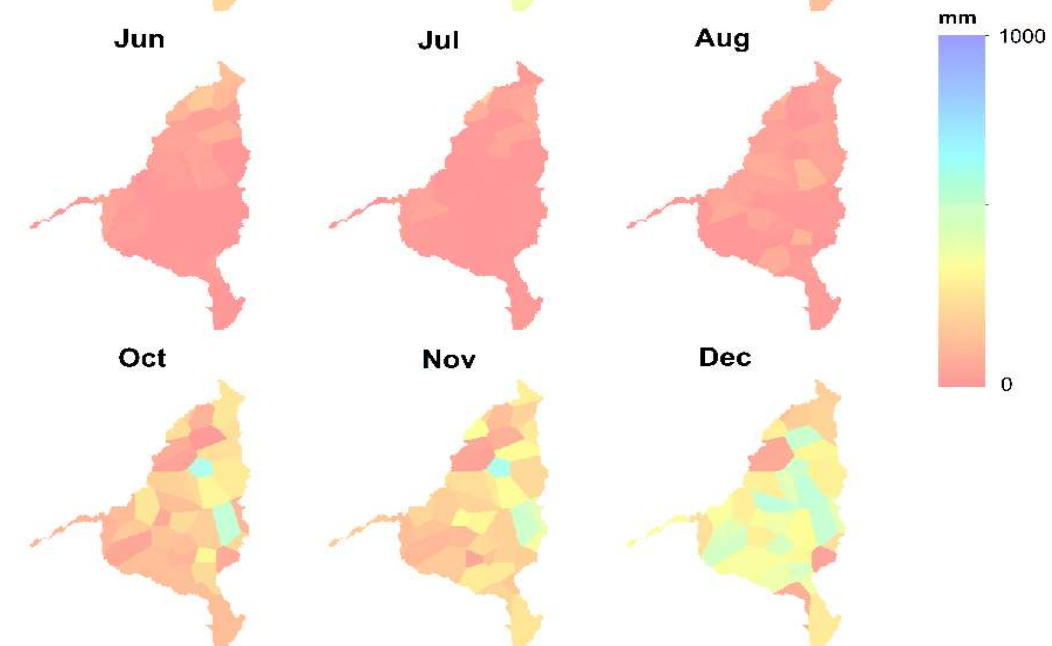

Figura 7 - Chuvas mensais (mm) interpoladas por polígonos de Thiessen definidos com base nas estações de treinamento para a bacia do rio Madeira. 
Dentre todos os procedimentos analisados, observou-se que o VN (Figura 5) não foi capaz de representar toda a área de estudo devido ao fato de que a distribuição espacial das estações pluviométricas não abrange toda a área da bacia para esse método. Por outro lado, nas zonas de valores interpolados, encontravam-se as estações de validação, possibilitando, assim, a realização dos cálculos.

As superfícies interpoladas geradas pelos métodos analisados (Figura 3, Figura, 4, Figura 5 e Figura 6) apresentaram diferenças significativas em magnitude, todavia as tendências de conformação da superfície pluviométrica foram similares, ou seja, as áreas de maiores e menores precipitações foram espacialmente semelhantes. Os padrões de precipitações mais extremas foram observados na porção nordeste a norte-nordeste da bacia, enquanto os menores índices foram encontrados na parte este-sudoeste até sul-sudoeste, localização da nascente do rio principal.

Dentre os métodos analisados, o BRM (Figura 6) mostrou-se o de menor eficiência, visto que gerou zonas de chuva negativa, contrapondo-se, dessa forma, à natureza do fenômeno que não pode apresentar tais valores.

O método inverso de distância ponderada (Figura 3) e vizinho natural (Figura 5) apresentaram certas particularidades, com a geração de linhas limítrofes significativas ao redor das estações pluviométricas utilizadas. Tal fenômeno também foi observado por Righi e Basso (2016) para a região da bacia do rio Piratinim na bacia do Madeira. Esses autores atribuíram tal tendência ao caráter estatístico dos métodos empregados, gerado pela influência de cada ponto na delimitação de um raio de ação de forma similar em todas as direções, o que possibilita a formação de círculos de influência.

O KG e o IDP apresentaram valores bem próximos todos os meses, estando dentro das magnitudes observadas pelos polígonos de Thiessen e média aritmética, porém a interpolação do KG em meses de transição de estações, abril e outubro, gerou campos de precipitação menos homogêneos e isoietas menos suavizadas.

O procedimento dos polígonos de Thiessen (Figura 7) apresentou transição espacial brusca, como era esperado pelo método que trabalha com segmentos de reta configurados por meio de mediatrizes definidas a partir das relações lineares de variação da chuva estabelecidas entre as estações de análise, delimitando-se, por conseguinte, as correspondentes áreas de influência. Os meses de abril e outubro foram os mais diferenciados em termos de distribuição pluviométrica ao longo da bacia. 
As médias aritméticas mensais estão discriminadas na Tabela 1.

Tabela 1. Média aritmética mensal $(\mathrm{mm})$ para a bacia do rio Madeira.

\begin{tabular}{cc}
\hline Mês & Média Aritmética Mensal \\
\hline Jan & 323,44 \\
Fev & 334,23 \\
Mar & 277,72 \\
Abr & 244,71 \\
Mai & 66,68 \\
Jun & 28,27 \\
\hline
\end{tabular}

\begin{tabular}{cc}
\hline Mês & Média Aritmética Mensal \\
\hline Jul & 17,87 \\
Ago & 34,53 \\
Set & 82,23 \\
Out & 181,90 \\
Nov & 230,80 \\
Dez & 325,47 \\
\hline
\end{tabular}

As médias seguiram as tendências do clima da região, que é caracterizado por precipitações mais intensas na estação chuvosa, compreendida entre os meses de novembro a março, enquanto, na estação seca, que abrange os meses de julho a setembro, a precipitação tem os menores valores registrados. Entre esses períodos, é possível identificar as fases de transição, como a existente da estação seca para a úmida e vice-versa, onde a precipitação aumenta ou reduz no decorrer dos meses.

A análise dos resíduos (Figura 8) ao longo dos meses mostrou que todas as técnicas empregadas no estudo tenderam a acertar de forma mais eficiente nos meses mais secos e com pouca pluviosidade. Por outro lado, os meses chuvosos apresentaram resíduos de maior magnitude, principalmente dezembro e janeiro, não tendo sido percebida, para esses dois meses, uma tendência de subestimação ou superestimação por parte de qualquer uma das técnicas analisadas. Todavia, nos meses de junho, julho e agosto, houve algum grau de superestimativa em todas os procedimentos, visto que os resíduos tenderam a ser positivos.
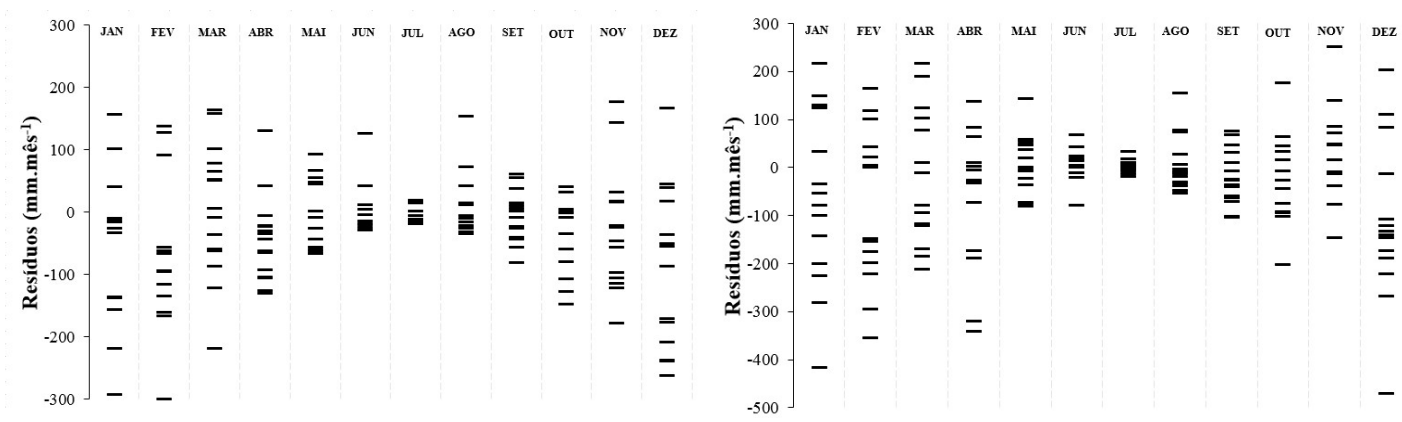

a)

b) 

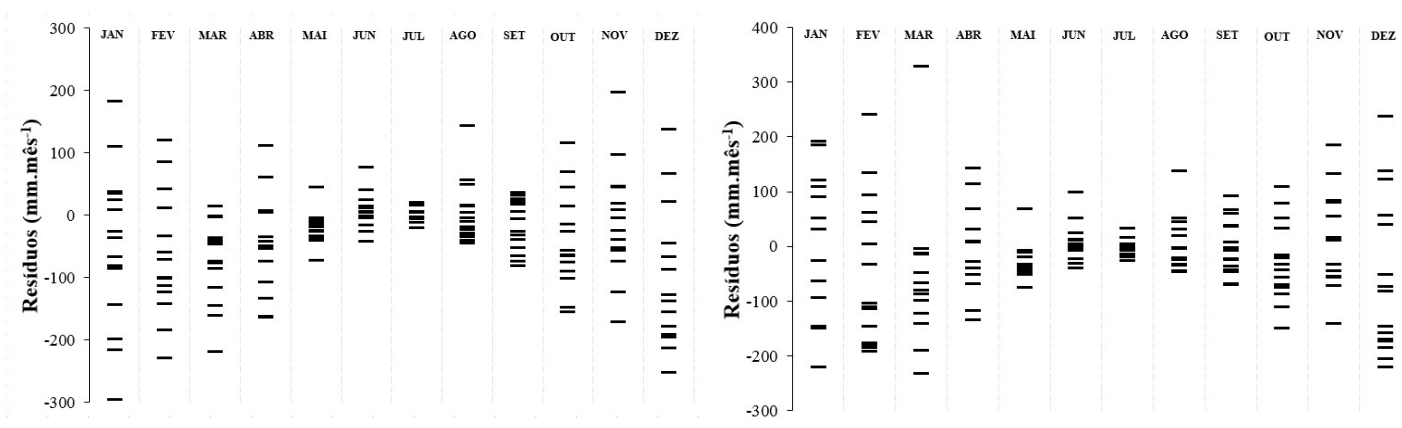

c)

d)
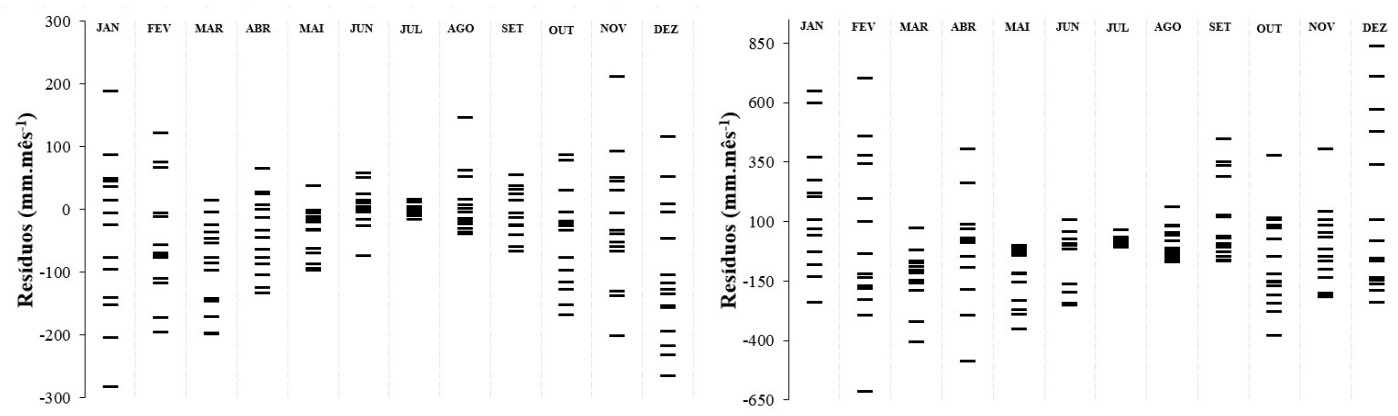

e)

f)

Figura 8. Resíduos mensais (mm) na bacia do rio Madeira: a) MA; b) PT; c) IDP; d) VN; e) $\mathrm{KG}$; f) BRM.

Cabe destacar que as menores amplitudes de resíduos foram observadas nas técnicas de IDP e de KG, denotando, assim, que essas técnicas tenderam a errar menos na estimativa espacial da chuva para a bacia do rio Madeira. Porém a média aritmética não teve valores muito distantes aos desses dois operadores, confirmando que, por vezes, uma técnica de estimação mais simples pode apresentar, em determinados casos, tendência de produzir resultados bastante similares em contraste com aqueles produzidos por interpoladores teoricamente mais sofisticados.

No entanto, ressalta-se que, dentre todas as técnicas, o BRM apresentou magnitudes residuais muito superiores, tanto negativamente quando positivamente para todos os meses, quando comparadas com todas as outras técnicas, indicando que é bastante limitado em termos de produzir um campo espacial de chuvas para a bacia hidrográfica estudada.

Os resultados das correlações mensais das técnicas (Tabela 2) seguiram as mesmas constatações da análise de resíduos. Os meses em que a correlação se apresentou mais alta 
foram os meses de seca, chegando a ser registrado uma correlação máxima de 0,81 no mês de julho para a técnica do KG.

Tabela 2. Coeficiente de correlação para os métodos de interpolação espacial empregados no estudo na escala mensal ao longo do ano de 2011 na bacia do rio Madeira.

\begin{tabular}{ccccccc}
\hline \multirow{2}{*}{ Mês } & \multicolumn{6}{c}{ Coeficiente de correlação (r) } \\
\cline { 2 - 7 } & MA & PT & IDP & VN & KG & BRM \\
\hline Janeiro & 0,00 & $-0,07$ & 0,23 & 0,44 & 0,28 & 0,30 \\
Fevereiro & 0,00 & 0,07 & 0,51 & 0,34 & 0,60 & 0,34 \\
Março & 0,00 & 0,34 & 0,44 & 0,18 & 0,32 & 0,17 \\
Abril & 0,00 & $-0,10$ & 0,16 & 0,26 & 0,44 & 0,14 \\
Maio & 0,00 & 0,49 & 0,79 & 0,71 & 0,61 & $-0,16$ \\
Junho & 0,00 & 0,67 & 0,75 & 0,56 & 0,68 & 0,02 \\
Julho & 0,00 & 0,69 & 0,71 & 0,34 & 0,81 & 0,08 \\
Agosto & 0,00 & $-0,09$ & 0,30 & 0,34 & 0,45 & $-0,17$ \\
Setembro & 0,00 & 0,05 & 0,30 & $-0,12$ & 0,39 & $-0,18$ \\
Outubro & 0,00 & 0,13 & $-0,05$ & 0,31 & $-0,24$ & $-0,40$ \\
Novembro & 0,00 & 0,43 & 0,46 & 0,47 & 0,27 & 0,24 \\
Dezembro & 0,00 & 0,32 & 0,49 & 0,22 & 0,51 & 0,20 \\
\hline
\end{tabular}

Os piores estimadores, segundo o parâmetro do coeficiente de correlação mensal, foram o BRM e o PT, excluindo-se, dessa análise, a média aritmética, que, devido ao fato de ser um valor constante, limita a formulação dessa medida de correlação apresentar um resultado relevante. O IDP e o KG, em todos os meses, mostram-se superiores em relação aos demais procedimentos, contudo, no mês de outubro, o KG apresentou uma melhor correlação em relação ao IDP, mesmo essa sendo negativa, o que indica que a técnica subestima o valor observado.

O efeito de correlações negativas em relação à aplicação de interpoladores e aos eventos de chuva também foi observado no estudo de Righi e Basso (2016), onde se observaram magnitudes de $-0,81,-0,80,-0,50$ e $-0,16$ para os interpoladores $B R M$, IDP, KG e VN, respectivamente.

Os índices de eficiência EMM, $\mathrm{r}^{2}$ e d estão na Tabela 3. 
Tabela 3. Análise de eficiência dos métodos de interpolação espacial empregados no estudo na bacia do rio Madeira.

\begin{tabular}{lccc}
\hline Método & EMM & $\mathbf{r}^{2}$ & D \\
\hline MA & 68,65 & 0,56 & 0,84 \\
PT & 83,31 & 0,46 & 0,79 \\
IDP & 63,64 & 0,63 & 0,87 \\
VN & 68,74 & 0,55 & 0,85 \\
KG & 62,71 & 0,63 & 0,87 \\
BRM & 144,20 & 0,14 & 0,48 \\
\hline
\end{tabular}

O cálculo do índice de concordância possibilitou avaliar a aderência dos valores de precipitação obtidos pelos interpoladores em comparação com os valores reais da precipitação média para a série histórica analisada. Esse índice varia de zero (não existe concordância) a "um" (concordância perfeita). Assim, observando os valores obtidos, nota-se que tanto o IDP quanto o KG obtiveram os melhores resultados, ambos 0,87 . Por outro lado, a média aritmética também apresentou uma magnitude próxima de 0,84, indicando que essa técnica é tão eficaz, no caso estudado, quanto o uso de interpoladores. Já a metodologia BRM obteve o menor grau do índice de concordância, cerca de 0,48 , mostrando novamente o quão limitado é o uso desse interpolador na bacia.

Os valores do coeficiente de determinação $\left(\mathrm{r}^{2}\right)$ seguiu a mesma lógica do índice de concordância, destacando que, tanto para o IDP quanto para o KG, 63\% na variação dos dados dos eventos observados podem ser explicados pela variação nos dados desses interpoladores. Em terceiro lugar, a média aritmética (MA) novamente mostrou-se mais eficaz em predizer o valor dos postos de validação, visto que o $\mathrm{r}^{2}$ desse procedimento foi de 0,55 , sendo ele maior que os métodos dos polígonos de Thiessen, do VN e do BRM.

Os valores dos erros modulares médios estiveram bem próximos para as técnicas $\mathrm{KG}$, VN, IDP e MA, que tiveram as menores magnitudes observadas. Reforça-se que a média aritmética pode ser aplicada na bacia hidrográfica em estudo com pouco prejuízo em relação à aplicação de técnicas mais sofisticadas de interpolação, o que pode reduzir o custo computacional associado, por exemplo, a atividades de preenchimento de falhas de estações pluviométricas. 
O BRM obteve o maior erro modular médio, concordando, assim, com os resultados anteriores que apontam a inadequação da aplicação desse interpolador de chuva para a bacia do rio Madeira. Essa constatação vai de encontro ao levantamento feito no trabalho de Magalhães et al. (2013) para o norte do Espirito Santo, que observou que tal método é satisfatório para interpolação espacial da precipitação anual, com um índice de concordância igual ao obtido pelo método de melhor desempenho do supracitado estudo, o IDP, e um erro quadrado médio bem reduzido.

De forma geral, o KG e IDP apresentaram a maior eficiência em todos os testes empregados no presente estudo. Os resultados similares desses interpoladores podem ser explicados pelo fato de que ambos possuem bases conceituais semelhantes, diferindo apenas nos pesos obtidos no procedimento de interpolação espacial com base na forma do semivariograma (MIRANDA et al., 2009). O desempenho próximo dessas duas técnicas também foi notado no estudo de Gardiman Junior et al. (2012) para a bacia do rio Itapemirim (ES).

O método de krigagem também é citado por Silva et al. (2011), por exemplo, como um método padrão para interpolação de precipitação pluvial nas regiões do Espírito Santo, apresentando índices estatístico satisfatórios.

$\mathrm{O}$ VN apresentou restrições espaciais de uso na bacia e não foi tão eficaz em contraste com as outras metodologias, contudo Righi e Basso (2016) apontaram essa técnica como o melhor interpolador testado para eventos pluviométricos anuais. Dessa forma, a limitação da aplicação desse procedimento na bacia pode residir na configuração da distribuição das estações testadas, indicando, assim, que o dimensionamento da rede pluviométrica na bacia do Madeira necessita ser revista para torná-la mais representativa.

\section{Considerações Finais}

Os métodos de interpolação empregados para a representação espacial da precipitação, a partir dos postos pluviométricos, para a análise da série histórica e para o ano de 2011, apresentaram, em alguns casos, valores negativos na correlação. Entre os métodos aplicados, aqueles que apresentaram maiores valores de correlação foram o método de krigagem (KG) e o inverso de distância ponderada (IDP), ainda que valor negativo desse indicador tenha sido obtido no que concerne ao mês de outubro para ambos os procedimentos. 
O método da função de base radial multiquadrada (BRM) obteve o pior resultado quando comparado com os demais métodos aplicados, mostrando-se inadequado ou pouco representativo para a bacia hidrográfica do rio Madeira, visto que apresentou significativas magnitudes negativas de chuva, o que é algo impossível de ocorrer.

A configuração espacial das estações presentes na bacia não permitiu aplicar a técnica do vizinho natural (VN) em toda a sua extensão, observando-se, assim, que a rede pluviométrica da bacia do rio Madeira não está suficientemente bem disposta espacialmente para captar a variabilidade da chuva na região.

No presente estudo, cabe destacar que a média aritmética das estações constituiu-se, no momento, em uma alternativa potencial e de baixo custo computacional no que tange ao preenchimento de dados faltantes em um posto pluviométrico bem como em implementação de estudos que comportem balanços hídricos simplificados na escala da bacia.

Por outro lado, na medida em que a média torna-se uma medida até certo ponto equivalente a outros procedimentos mais sofisticados de interpolação espacial, com desempenho similar, pode-se estar diante de duas faces da mesma moeda.

De um lado, poder-se-ia cogitar de que há uma rede suficientemente densa de postos de medição, de tal forma que a média é representativa do valor a ser empregado em um balanço hídrico agregado méido na escala de uma bacia hidrográfica, o que definitivamente não é o caso. Complementarmente, no caso de preenchimento de falhas, o simples uso da média como recomendação revela que não foi possível agregar outras informações sobre o fenômeno da chuva na explicação do seu comportamento.

De outro, o presente estudo explicita que a rede de postos pluviométricos necessita ser acrescida de novas estações que efetivamente agreguem informação. Em outras palavras, há limitação severa em termos de medição da chuva a partir da atual rede pluviométrica instalada na bacia do rio Madeira.

Nesse sentido, a recomendação final do estudo é de que investigações futuras concentrem-se na avaliação do conteúdo da informação da rede pluviométrica atual e do seu correspondente valor intrínseco, de forma que se possa melhor balizar a sua revisão e implantação de novas estações de medição observacional local.

A recomendação alia-se ao fato de que dados de chuva por satélite podem ser também inclusos na análise proposta de reavaliação da rede de medições in situ com vistas ao emprego em estudos integrados espaço-temporais do fenômeno pluviométrico. Os reflexos da 
recomendação têm impacto na escala mensal bem como nas escalas diária e horária, onde captar a variabilidade espaço-temporal assume importância crescente em estudos diversos.

\section{AGRADECIMENTOS}

Os autores agradecem a bolsa de estudos concedida ao primeiro autor fornecida pelo CNPq - Conselho Nacional de Desenvolvimento Científico e Tecnológico. Registra-se, também, que o presente trabalho foi realizado com apoio da Coordenação de Aperfeiçoamento de Pessoal de Nível Superior - Brasil (CAPES) - Código de Financiamento 001. Agradecimentos são estendidos à FAPERJ, por meio do projeto FAPERJ - Pensa Rio - Edital 34/2014 (2014-2021) - E-26/010.002980/2014, bem como ao suporte oferecido pelo CNPq por meio do projeto Edital $n^{\circ}$ 06/2019 - Processo 303846/2019-4 e projeto Edital Universal $n^{\circ}$ 28/2018 - Processo 435714/2018-0.

\section{REFERÊNCIAS BIBLIOGRÁFICAS}

ALMEIDA, A. Q.; RIBEIRO, A.; PAIVA, Y. G.; RASCON, N. J. L.; LIMA, E. P. Geoestatística no estudo de modelagem temporal da precipitação. Revista Brasileira de Engenharia Agrícola e Ambiental, v. 15, n. 4, p. 354-358, 2011.

ANDRADE, C. D. Análise pluviométrica da bacia transfronteiriça do Rio Madeira. 2008. Dissertação (Mestrado em Engenharia Civil) - Instituto Alberto Luiz Coimbra de pósgraduação e pesquisa de engenharia, Universidade Federal do Rio de Janeiro, Rio de Janeiro.

CLARKE, R. T.; DIAS, P. L. S. As necessidades de observação e monitoramento dos ambientes brasileiros quanto aos recursos hídricos - versão 1.0 (preliminar). Brasília, CGEE - Centro de Gestão e Estudos Estratégicos, CT-Hidro - Fundo Setorial de Recursos Hídricos, 41p. 2003.

CONTI, G. N. Estimativa da precipitação através de técnicas de sensoriamento remoto: Estudo de caso para o estado do Rio Grande do Sul. Dissertação (Mestrado em Recursos Hídricos), IPH, Universidade Federal do Rio Grande do Sul, 2002. 
ESPINOZA, J. C., RONCHAIL, J., GUYOT, J. L., COCHENEAU, G., FILIZOLA, N., LAVADO, W., DE OLIVEIRA, E., POMBOSA, R., VAUCHEL, P. Spatio - Temporal rainfall variability in the Amazon Basin Countries (Brazil, Peru, Bolivia, Colombia and Ecuador). International Journal of Climatology, 29, 1574-1594, 2009.

ESRI.. ArcGIS Desktop: release 10.2. [Versão de teste] Redlands, CA: Environmental Systems Research Institute, 2017.

GARDIMAN JUNIOR, B. S.; MAGALHÃES, I. A. L.; FREITAS, C. A. A.; CECÍLIO, R. A.. Análise de técnicas de interpolação para espacialização da precipitação pluvial na bacia do rio Itapemirim (ES). Revista do Setor de Ciências Agrárias e Ambientais, v. 8, n.1, p. 61-71, 2012.

KOHAVI, R. A study of cross-validation and bootstrap for accuracy estimation and model selection. In: International joint Conference on artificial intelligence. [S.l.: s.n.]. v. 14, p. 1137-1145, 1995.

MAGALHÃES, I. A. L.; ALMEIDA, K. L.; THIAGO, C. R. L.; GARDIMAN JUNIOR, B. S.; ZANETTI, S. S.; CECÍLIO, R. A. Análise de métodos de interpolação para espacialização da precipitação pluvial na região norte do estado do Espírito Santo. In: Simpósio Brasileiro de Sensoriamento Remoto, XVI, Foz do Iguaçu, Paraná, 2013. Anais... Foz do Iguaçu: SBSR, $2013,1-8$.

MIRANDA, F. A.; FREITAS, S. R. C.; FAGGION, P. L. Integração e interpolação de dados de anomalias ar livre utilizando-se a técnica de RNA e krigagem. Boletim de Ciências Geodésicas, v.15, p.428-433, 2009.

NOBRE, C. A.; OBREGÓN, G. O.; MARENGO, J. A.; FU, R.; POUEDA, G. Características do Clima Amazônico: Aspectos Principais. In: Amazonia and Global Change, Geophysical Monograph Series, American Geophysical Union, p 49-162. 2009. 
RIGHI, E.; BASSO, L. A. Application and analysis of interpolation techniques for spatialization of rainfall. Ambiência (UNICENTRO), v. 12, p. 101-117, 2016.

SILVA, K. R.; CECÍlIO, R. A.; XAVIER, A. C.; PEZZOPANE, J. R. M.; GARCIA, G. O. Interpolação Espacial da Precipitação no Estado do Espírito Santo. Revista Floresta e Ambiente, v. 18, n. 4, p. 417-427, 2011

STRECK, N. A.; BURIOL, G. A.; HELDWEIN, A. B.; GABRIEL, L. F.; PAULA, G. M. de. Associação da variabilidade da precipitação pluvial em Santa Maria com a oscilação decadal do Pacífico. Pesquisa Agropecuária Brasileira, v.44, n.12, p.1553-1561, 2009.

TUCCI, C. E. M. (Org.). Hidrologia. Ciências e aplicação. 4. Ed. Porto Alegre: Ed. da Universidade: ABRH: EDUSP, 2009. 